Cristina L. S. Sisinno 1

Annibal D. Pereira Netto 2

Eliane Cristina P. do Rego 3

Guilherme dos Santos V. Lima 3

\section{Hidrocarbonetos policíclicos aromáticos em resíduos sólidos industriais: uma avaliação preliminar do risco potencial de contaminação ambiental e humana em áreas de disposição de resíduos}

\author{
Polycyclic aromatic hydrocarbons in industrial \\ solid waste: a preliminary evaluation of the \\ potential risk of environmental and human \\ contamination in waste disposal areas
}

1 Centro de Estudos da Saúde do Trabalhador e Ecologia Humana, Escola Nacional de Saúde Pública. Fundação Oswaldo Cruz. Rua Leopoldo Bulhões 1480, Rio de Janeiro, $R J$ 21041-210, Brasil. sisinno@ensp.fiocruz.br 2 Departamento de Química Analítica, Instituto de Química, Universidade Federal Fluminense. Outeiro de São João Batista $s / n$, Campus do Valonguinho, Niterói, RJ 24020-150, Brasil.

3 Centro de Tecnologia Ambiental, Federação das Indústrias do Estado do Rio de Janeiro. Rua Morais e Silva 53, Rio de Janeiro, $R J$ 20271-030, Brasil.

\begin{abstract}
Proper solid waste disposal is important to avoid human and environmental contamination. The NBR 10,004 Waste Classification lists several polycyclic aromatic hydrocarbons (PAHs) and indicates that the presence of at least one PAH in a waste sample is enough to classify it as hazardous. The aim of this study was a preliminary evaluation of PAHs in solid waste samples from selected industries to obtain a preliminary overview of their potential for contamination in case of improper disposal. One or more PAHs listed in NBR 10,004 (benzo[a]anthracene, benzo[a]pyrene, benzo[b]fluoranthene, benzo[k]fluoranthene, indene[1,2,3-c,d]pyrene, chrysene, or fluoranthene) were found in all samples, thus leading to their classification as hazardous waste. Our results showed that toxicologically relevant PAHs were found in all the samples, indicating that their final disposal must be performed in appropriate areas in order to minimize human health risks and environmental contamination from waste disposal areas.

Key words Hazardous Waste; Terminal Disposal of Chemical Waste; Polycyclic Aromatic Hydrocarbons; Waste Classification
\end{abstract}

Resumo A disposição adequada dos resíduos sólidos é importante para evitar que os mesmos se transformem em fonte de contaminação ambiental e humana. A NBR 10.004 - Classificação de Resíduos - lista vários hidrocarbonetos policíclicos aromáticos (HPAs) e indica que, a presença de pelo menos um deles na massa bruta do resíduo é suficiente para classificá-lo como resíduo perigoso. O objetivo deste estudo foi verificar a presença de HPAs em amostras de resíduos sólidos provenientes de alguns segmentos industriais, para se obter uma avaliação preliminar do potencial de contaminação que estes resíduos podem representar, caso não recebam destino adequado. Pelo menos um dos HPAs previstos na NBR 10.004 (benzo[a]antraceno, benzo[a]pireno, benzo[b]fluoranteno, benzo[k]fluoranteno, indeno[1,2,3-c,d]pireno, criseno ou fluoranteno) foi identificado em todos os resíduos estudados, classificando-os como perigosos. Nossos resultados indicam que todos os resíduos estudados continham HPAs de importância toxicológica, o que implica que sua disposição final seja feita em locais adequados para minimizar os riscos à saúde humana e ambiental oriundos das áreas de disposição de resíduos.

Palavras-chave Resíduos Perigosos; Disposição Final de Resíduos Químicos; Hidrocarbonetos Policíclicos Aromáticos; Classificação de Resíduos 


\section{Introdução}

Hidrocarbonetos policíclicos aromáticos (HPAs) são poluentes orgânicos de importância ambiental e interesse toxicológico, pois muitos apresentam propriedades pré-carcinogênicas e/ou mutagênicas para homens e animais (Boffetta et al., 1997; Pereira Netto et al., 2000; WHO, 1988). Os mecanismos de carcinogênese destas substâncias foram revistos recentemente e, de um modo geral, vários órgãos podem ser afetados (WHO, 1988). Há relação com o mecanismo de carcinogênese e a estrutura molecular dos HPAs que, portanto, têm potencial carcinogênico diferenciado. A International Agency for Research on Cancer (IARC, 2002) estabeleceu uma classificação de HPAs e de alguns materiais que os contêm, conforme os dados disponíveis sobre a carcinogenicidade destes materiais ou substâncias (Tabela 1).

HPAs são emitidos por fontes naturais (vulcões, por exemplo) ou antropogênicas (derramamento de petróleo, queima de combustíveis, resíduos industriais, etc.) (Lopes \& Andrade, 1996; Vo Dinh et al., 1998) e muitos processos térmicos dão origem a essas substâncias. Após a emissão, os HPAs, que são substâncias lipofílicas e podem ter grande persistência no meio ambiente, distribuem-se nos compartimentos ambientais em proporções que dependem de suas propriedades físico-químicas e das características de cada compartimento ambiental. A Environmental Protection Agency (EPA) estabeleceu uma lista de 16 HPAs considerados prioritários para monitoramento ambiental, em função de sua carcinogenicidade e ocorrência (Tabela 1).

A ingestão de HPAs por seres humanos ocorre por diversas vias como a inalação de ar, a ingestão de águas, solos, poeiras e alimentos, contato através da pele etc., que têm importância relativa diferente (WHO, 1988).

Em resíduos sólidos a presença de HPAs é de interesse, uma vez que pode ocorrer contaminação humana direta - por contato com o resíduo (no manuseio, no tratamento ou na disposição) ou indireta - causada pelo destino final inadequado dos resíduos e conseqüente contaminação ambiental do solo, lençóis freáticos, corpos d'água superficiais, biota e ar.

A NBR 10.004 - resíduos sólidos - classificação (ABNT, 1987b), tem por objetivo classificar os resíduos sólidos quanto aos seus riscos potenciais ao meio ambiente e à saúde pública. Esta classificação é estabelecida em função das substâncias neles identificadas e em testes laboratoriais complementares, onde vários parâmetros químicos são analisados na massa bruta e nos extratos lixiviados e solubilizados dos resíduos. Três categorias são previstas na norma: classe I - resíduos perigosos; classe II - resíduos não-inertes; classe III - resíduos inertes. Esta classificação é fundamental para o gerenciamento adequado dos resíduos uma vez que possibilita a determinação do seu correto manuseio, transporte, armazenamento e tratamento ou destinação final.

$\mathrm{O}$ anexo D - listagem no 4 da NBR 10.004 (ABNT, 1987b), relaciona substâncias e espécies químicas que conferem periculosidade aos resíduos. Dentre estas, estão incluídos diversos HPAs como benzo[a]pireno, fluoranteno, benzo[a] antraceno, benzo[b]fluoranteno, benzo[k] fluoranteno, criseno etc. (Tabela 1). A presença de um desses compostos no resíduo é suficiente para classificá-lo como resíduo perigoso (classe I).

Dessa forma, a identificação desses HPAs em resíduos é de relevante interesse para a saúde pública, uma vez que a classificação de um resíduo como perigoso implica a necessidade de formas adequadas de gerenciamento para evitar que ocorra contaminação ambiental e humana.

Segundo estimativas da Fundação Estadual de Engenharia do Meio Ambiente (FEEMA, 2000), órgão de fiscalização ambiental do Estado do Rio de Janeiro, do total de aproximadamente 500 mil toneladas de resíduos industriais produzidos mensalmente pelas indústrias situadas no Estado, a maior parte constitui-se de resíduos não-inertes (classe II), seguido de resíduos inertes (classe III) e, por fim, de resíduos perigosos (classe I). Cerca de $20 \mathrm{mil}$ toneladas de resíduos perigosos são produzidos no Estado por mês, correspondendo a óleo usado, resíduos contendo metais pesados, solventes, resíduos provenientes do re-refino de óleos usados e resíduos orgânicos gerados em plantas de refino de petróleo.

As principais formas de tratamento e destinação final dos resíduos produzidos no Estado do Rio de Janeiro são: reciclagem, aterro municipal, co-processamento, aterro industrial, estocagem, incineração, incorporação, fertilização ou landfarming e aterro de terceiros. A destinação de resíduos perigosos ocorre principalmente por meio de reciclagem ou por deposição em aterros municipais e industriais. Observa-se que muitos dos resíduos industriais perigosos chegam aos vazadouros de lixo misturados com os resíduos industriais comuns (FEEMA, 2000).

Os aterros municipais são geralmente representados por aterros controlados ou vazadouros de lixo, cuja infra-estrutura não é ade- 
quada para a destinação de resíduos com características de periculosidade. Desse modo, pelo menos parte de substâncias consideradas perigosas (dentre elas, alguns HPAs) presentes nos resíduos, tem como destino final o solo desses aterros, podendo ter como possíveis conseqüências, a contaminação ambiental e humana por substâncias carcinogênicas.

Essa hipótese é coerente com os resultados recentes que demonstraram a presença de HPAs em concentrações de 0,02 a $4,2 \mu \mathrm{g} / \mathrm{L}$ em chorume proveniente do Aterro Controlado do Morro do Céu, em Niterói, Rio de Janeiro (Pereira Netto et al., 2002). Apesar de haver outras fontes de HPAs e de compostos orgânicos em depósitos de resíduos (Öman \& Hynning, 1993), incluindo deposição atmosférica (Ahel \& Tepic, 2000; Wild \& Jones, 1995) e focos espontâneos de incêndio nestas áreas devido a presença de altas concentrações de metano (Ruokojarvi et al., 1995), a introdução dessas substâncias por meio de resíduos deve ser considerada.

O objetivo deste trabalho foi avaliar a presença de HPAs, incluindo os previstos na NBR 10.004, em amostras de resíduos sólidos provenientes de alguns segmentos industriais, a fim de obter um quadro preliminar do potencial de contaminação que estes resíduos podem representar, caso não recebam destinação adequada.

\section{Materiais e métodos}

HPAs fornecidos pela Aldrich Chemical Co. (Wisconsin, Estados Unidos) e pela AccuStandard (Connecticut, Estados Unidos) foram usados como padrões. Hexano e tolueno (Omnisolv - Residue Analysis - E. M. Science, New Jersey, Estados Unidos) e diclorometano (Absolv - Tedia, Rio de Janeiro, Brasil) foram empregados na preparação dos extratos.

Sete amostras (lodos, solo contaminado e óleo queimado) de resíduos provenientes de indústrias localizadas no Estado do Rio de Janeiro, e de diferentes segmentos (petroquímico, metalmecânica e de bebidas) foram coletadas segundo a metodologia recomendada pela NBR 10.007 - amostragem de resíduos (ABNT, 1987a). As indústrias em questão tinham interesse em analisar seus resíduos de acordo com a NBR 10.004, a fim de classificá-los segundo as normas brasileiras.

Alíquotas de 0,5 a $2 \mathrm{~g}$ das amostras foram pesadas ao décimo de mg e submetidas à extração em banho de ultra-som com 4 porções de $20 \mathrm{~mL}$ de diclorometano por 20 minutos cada. Os extratos de cada amostra foram combinados e após adição de $100 \mu \mathrm{L}$ de tolueno, con-
Tabela 1

Classificação dos HPAs estudados quanto a sua carcinogenicidade (IARC), carcinogenicidade e ocorrência (EPA) e periculosidade (ABNT).

\begin{tabular}{lccc}
\hline HPAs & IARC & EPA & ABNT \\
\hline Fluoreno & 3 & $\mathrm{P}$ & $\mathrm{NM}$ \\
Fenatreno & 3 & $\mathrm{P}$ & $\mathrm{NM}$ \\
Antraceno & 3 & $\mathrm{P}$ & $\mathrm{NM}$ \\
Metilfenantrenos+Metilantracenos & 3 & $\mathrm{P}$ & $\mathrm{NM}$ \\
Pireno & 3 & $\mathrm{P}$ & $\mathrm{NM}$ \\
Fluoranteno & 3 & $\mathrm{P}$ & $\mathrm{CP}$ \\
Benzo[a]antraceno & $\mathrm{P}$ & $\mathrm{P}$ & $\mathrm{CP}$ \\
Criseno & 3 & $\mathrm{P}$ & $\mathrm{CP}$ \\
Benzo[b]fluoranteno & $\mathrm{P}$ & $\mathrm{CP}$ \\
Benzo[k]fluoranteno & $\mathrm{B}$ & $\mathrm{P}$ & $\mathrm{NM}$ \\
Benzo[e]pireno & 2B & $\mathrm{P}$ & $\mathrm{NM}$ \\
Benzo[a]pireno & 3 & $\mathrm{P}$ & $\mathrm{CP}$ \\
Indeno[1,2,3-c,d]pireno & 2A & $\mathrm{P}$ & $\mathrm{CP}$ \\
Dibenzo[a,h]antraceno & 2B & $\mathrm{P}$ & $\mathrm{CP}$ \\
Benzo[g,h,i]perileno & 2A & $\mathrm{P}$ & $\mathrm{NM}$ \\
Coroneno & 3 & $\mathrm{P}$ & $\mathrm{NM}$
\end{tabular}

IARC = International Agency for Research on Cancer; EPA = Environmental Protection Agency; ABNT = Associação Brasileira de Normas Técnicas (NBR 10.004).

$2 A=$ Provável carcinogênico para humanos - limitada evidência em humanos e suficiente em animais (IARC); $2 B=$ Possivel carcinogênico para humanos limitada evidência em humanos e insuficiente em animais (IARC); 3 = Não é classificado como carcinogênico para humanos (IARC); $P=$ Prioritário (EPA); $\mathrm{CP}=$ Confere periculosidade $(\mathrm{ABNT}) ; \mathrm{NM}=\mathrm{Não}$ mencionado no anexo $\mathrm{D}$, listagem no 4 (ABNT).

centrados em evaporador rotatório $\left(\mathrm{T}<40^{\circ} \mathrm{C}\right)$ até cerca de $10 \mathrm{~mL}$. O extrato foi centrifugado e o sobrenadante evaporado $\operatorname{com} \mathrm{N}_{2}$ de alta pureza até cerca de $500 \mathrm{~mL}$. O extrato concentrado foi submetido à limpeza em cartuchos de extração em fase sólida $\left(\mathrm{SiO}_{2} ; 3 \mathrm{~mL} ; 500 \mathrm{mg}\right)$ empregado-se um sistema de vácuo (J. T. Baker). A fração rica em HPAs foi eluída com hexano, concentrada com $\mathrm{N}_{2}$ e mantida em vial até a análise (Pereira Netto et al., 2001, 2002).

Os extratos foram analisados por cromatografia a gás de alta resolução acoplada à espectrometria de massas (CGAR-EM) em um cromatógrafo AutoSystem TurboMass (Perkin Elmer). Solução contendo 23 HPAs, incluindo os previstos na NBR 10.004 e os considerados prioritários pela EPA, foi usada na otimização das condições de separação cromatográficas em coluna DB5-ms $(30 \mathrm{~m} ; 0,25 \mathrm{~mm} ; 0,25 \mathrm{~mm})$. Os extratos foram analisados por varredura (50350u.m.a.) e por monitoramento seletivo de íons (SIM) com os íons de peso molecular (Tuominem et al., 1986). A identificação foi realizada combinando-se informações obtidas pelos tempos de retenção, por fragmentogramas de íons específicos e cromatogramas em SIM. 


\section{Resultados e discussão}

Os extratos das amostras analisadas apresentaram cromatogramas de corrente iônica total (TIC) diferentes, dependendo da origem das amostras. Alguns perfis cromatográficos eram bastante complexos, indicando a presença de grande número de substâncias nos extratos. Hidrocarbonetos alifáticos foram, de um modo geral, as substâncias predominantes nos extratos analisados.

Os resultados obtidos na análise dos diferentes resíduos estudados encontram-se na Tabela 2. Diversos HPAs (fluoreno, fenantreno, antraceno, metilfenantrenos+metilantracenos, pireno, fluoranteno, benzo[a]antraceno, criseno, benzo[b]fluoranteno, benzo[k]fluoranteno, benzo[e]pireno, benzo[a]pireno, indeno[1,2,3cd]pireno, dibenzo[a,h] antraceno, coroneno e benzo[g,h,i]perileno) foram identificados nas amostras analisadas.

Os resultados obtidos demonstram que todas as amostras apresentaram mais de um dos HPAs que conferem periculosidade aos resíduos. Dentre essas substâncias perigosas, descritas no anexo D da NBR 10.004, benzo[a]pireno, fluoranteno e criseno foram os HPAs identificados em todas as amostras estudadas.
O maior número de amostras estudadas foram provenientes do setor petroquímico (amostras 1, 2, 3, 5 e 6), que é um dos setores onde ocorre maior produção de resíduos contendo HPAs. Nessas amostras, foram sempre encontrados benzo[a]pireno, fluoranteno e criseno, que estão listados entre os HPAs que conferem periculosidade aos resíduos.

$\mathrm{Na}$ amostra de óleo queimado originada de caldeira (amostra 4) de uma indústria do setor alimentício e na de solo contaminado (amostra 7), proveniente de uma indústria desativada do setor metalmecânico (igualmente considerado um resíduo), foram encontrados todos os HPAs que conferem periculosidade a um resíduo, como benzo[a]pireno, fluoranteno, benzo[a] antraceno, benzo[b]fluoranteno, indeno[1,2,3c,d]pireno, dibenzo[a,h] antraceno e criseno.

A identificação desses HPAs nas amostras analisadas merece importância sob o aspecto ambiental e de saúde. A presença de benzo[a]pireno, benzo[a]antraceno e dibenzo[a,h] antraceno - classificados pelo IARC como prováveis carcinogênicos para seres humanos (2A) - e de benzo[b]fluoranteno, benzo[k]fluoranteno e indeno[1,2,3-c,d]pireno - considerados possíveis carcinogênicos (2B) - em alguns dos resíduos analisados, deve ser destacada pelo inte-

Tabela 2

HPAs estudados e identificados em cada amostra.

\begin{tabular}{|c|c|c|c|c|c|c|c|}
\hline HPAs & $\begin{array}{c}\text { Amostra } 1 \\
\text { (lodo) }\end{array}$ & $\begin{array}{c}\text { Amostra } 2 \\
\text { (lodo) }\end{array}$ & $\begin{array}{c}\text { Amostra } 3 \\
\text { (lodo) }\end{array}$ & $\begin{array}{l}\text { Amostra } 4 \\
\text { (óleo } \\
\text { queimado) }\end{array}$ & $\begin{array}{c}\text { Amostra } 5 \\
\text { (areia } \\
\text { contaminada } \\
\text { com óleo) }\end{array}$ & $\begin{array}{c}\text { Amostra } 6 \\
\text { (lodo) }\end{array}$ & $\begin{array}{c}\text { Amostra } 7 \\
\text { (solo de indústria } \\
\text { desativada) }\end{array}$ \\
\hline Fluoreno & - & + & + & + & + & + & + \\
\hline Fenatreno & + & + & + & + & + & + & + \\
\hline Antraceno & + & + & + & + & + & + & + \\
\hline $\begin{array}{l}\text { Metilfenantrenos + } \\
\text { Metilantracenos }\end{array}$ & + & NA & NA & + & NA & NA & + \\
\hline Pireno & + & + & + & + & + & + & + \\
\hline Fluoranteno & + & + & + & + & + & + & + \\
\hline Benzo[a]antraceno & + & + & + & + & - & + & + \\
\hline Criseno* & + & + & + & + & + & + & + \\
\hline Benzo[b]fluoranteno & + & + & + & + & - & + & + \\
\hline Benzo[k]fluoranteno & + & + & + & + & - & + & + \\
\hline Benzo[e]pireno & + & NA & NA & + & NA & NA & + \\
\hline Benzo[a]pireno & + & + & + & + & + & + & + \\
\hline Indeno[1,2,3-c,d]pireno & - & - & - & + & - & + & + \\
\hline Dibenzo[a,h]antraceno** & - & - & - & - & - & + & + \\
\hline Benzo[g,h,i]perileno & - & - & + & + & - & + & + \\
\hline Coroneno & - & NA & NA & + & NA & NA & + \\
\hline
\end{tabular}

(+) Presente; (-) Ausente; NA = Não analisado.

* coelui parcialmente com trifenileno;

** coelui com dibenzo[a,c]antraceno. 
resse que este fato representa para a saúde pública, uma vez que estas substâncias podem atingir seres humanos em qualquer uma das etapas de gerenciamento dos resíduos em questão.

O segundo aspecto de importância é que, com base nestes dados, todos os resíduos estudados podem receber a classificação de resíduos perigosos pela NBR 10.004, implicando o manuseio, transporte e destinação especiais. Também é fundamental observar que todos os resíduos apresentaram vários dos HPAs previstos na norma quando a presença de apenas um deles já seria suficiente para classificá-los como perigosos.

\section{Conclusões}

Diversos HPAs foram identificados nas amostras estudadas, sendo que benzo[a]pireno, fluoranteno e criseno foram identificados em todas elas. A presença destas substâncias confere periculosidade aos resíduos, fazendo com que haja a necessidade de um gerenciamento adequado dos mesmos. A presença de benzo[a]pireno nestas amostras deve ser destacada, pois este HPA é uma das substâncias sobre as quais se tem mais informações a respeito de suas propriedades pré-carcinogênicas.

\section{Agradecimentos}

C. L. S. Sisinno gostaria de expressar seus agradecimentos aos profissionais do Centro de Tecnologia Ambiental da Federação das Indústrias do Estado do Rio de Janeiro. A. D. Pereira Netto agradece à Fundação de Amparo à Pesquisa do Estado do Rio de Janeiro, cujo financiamento do Projeto E-26/170.827/2000 viabilizou indiretamente a execução deste trabalho e ao Conselho Nacional de Desenvolvimento Científico e Tecnológico (Projeto 478346/01-8).
Análises quantitativas destes resíduos estão em fase de implementação. Estes resultados permitirão avaliar a quantidade de HPAs introduzida nas áreas de disposição por resíduos industriais e estimar a quantidade destas substâncias que é deliberadamente introduzida no meio ambiente.

Nossos resultados indicam que é desejável e necessário que o gerenciamento adequado dos resíduos industriais seja seriamente considerado por todas as esferas envolvidas, inclusive pelo governo, setor normativo, setor fiscalizador e pelas próprias indústrias produtoras de resíduos perigosos, uma vez que todos os atores citados possuem responsabilidade sobre o quadro atual de degradação ambiental ocasionado pela disposição inadequada de resíduos.

Desse modo, um controle mais rigoroso e maiores opções de destino final para resíduos perigosos no Estado do Rio de Janeiro devem ser observados. Deve-se ressaltar finalmente, que a disposição inadequada de resíduos contendo HPAs representa uma fonte potencial de contaminação dos compartimentos ambientais e conseqüentemente, de seres humanos, principalmente se forem consideradas as quantidades de resíduos produzidas mensalmente e a natureza físico-química dessas substâncias (lipossolúveis e semivoláteis).

\section{Referências}

ABNT (Associação Brasileira de Normas Técnicas), 1987a. Amostragem de Resíduos - Procedimento NBR 10.007. Rio de Janeiro: ABNT.

ABNT (Associação Brasileira de Normas Técnicas), 1987b. Resíduos Sólidos - Classificação - NBR 10.004 Rio de Janeiro: ABNT.

AHEL, M. \& TEPIC, N., 2000. Distribution of polycyclic aromatic hydrocarbons in a municipal solid waste landfill and underlying soil. Bulletin of Environmental Contamination and Toxicology, 65:236-243.

BOFFETTA, P.; JOURENKOVA, N. \& GUSTAVSSON, P., 1997. Cancer risk from occupational and environmental exposure to polycyclic aromatic hydrocarbons. Cancer Causes \& Control, 8:444-472. 
FEEMA (Fundação Estadual de Engenharia do Meio Ambiente), 2000. Gestão de Resíduos - Relatório Semestral de Atividades do Programa de Despoluição da Baía de Guanabara - Setembro/2000. Rio de Janeiro: FEEMA. (mimeo.)

IARC (International Agency for Research on Cancer), 2002. Complete List of Agents, Mixtures and Exposures Evaluated and Their Classification. 3 May $2002<$ http://www.iarc.fr>.

LOPES, W. A. \& ANDRADE, J. B., 1996. Fonte, formação, reatividade e quantificação de hidrocarbonetos policíclicos aromáticos (HPA) na atmosfera. Química Nova, 19:497-516.

ÖMAN, C. \& HYNNING, P. A., 1993. Identification of organic compounds in municipal landfill leachates. Environmental Pollution, 80:265-271.

PEREIRA NETTO, A. D.; BARRETO, R. P.; MOREIRA, J. C. \& ARBILLA, G., 2001. Preliminary comparison of PAH in total suspended particulate samples taken at Niterói and Rio de Janeiro Cities, Brazil. Bulletin of Environmental Contamination and Toxicology, 66:36-43.

PEREIRA NETTO, A. D.; MOREIRA, J. C.; DIAS, A. E. X. O.; ARBILLA, G.; FERREIRA, L. F. V.; OLIVEIRA, A. S. \& BAREK, J., 2000. Avaliação da contaminação humana por hidrocarbonetos policíclicos aromáticos (HPAs) e seus derivados nitrados (NHPAs): Uma revisão metodológica. Química Nova, 23: 765-773.
PEREIRA NETTO, A. D.; SISINNO, C. L. S.; MOREIRA, J. C.; ARBILLA, G. \& DUFRAYER, M. C., 2002. Polycyclic aromatic hydrocarbons in leachate from a municipal solid waste dump of Niterói City, RJ, Brazil. Bulletin of Environmental Contamination and Toxicology, 68: 148-154.

RUOKOJARVI, P.; RUSKANEN, J.; ETTALA, M.; RAHKONEN, P. \& TARHANEN, J., 1995. Formation of polyaromatic hydrocarbons and polichlorinated organic compounds in municipal waste landfill fires. Chemosphere, 31:3899-3908.

TUOMINEN, J.; WICKSTRÖM, K. \& PYSALO, H., 1986. Determination of polycyclic aromatic compounds by GLC-selected ion monitoring (SIM) technique. Journal of High Resolution Chromatography Communication, 9:469-471.

VO DINH, T.; FETZER, J. \& CAMPIGLIA, A. D., 1998. Monitoring and characterization of polyaromatic compounds in the environment. Talanta, 47:943969.

WHO (World Health Organization), 1988. Selected Non-Heterocyclic Polycyclic Aromatic Hydrocarbons. IPCS (International Programme on Chemical Safety). Geneva: WHO.

WILD, S. R. \& JONES, K. C., 1995. Polynuclear aromatic hydrocarbons in the United Kingdom environment: A preliminary source inventory and budget. Environmental Pollution, 88:91-108.

Recebido em 11 de junho de 2002

Versão final reapresentada em 20 de agosto de 2002 Aprovado em 6 de novembro de 2002 